\title{
The Yang-Mills Collective-Coordinate Potential
}

\author{
John Lott \\ Department of Mathematics, Harvard University, Cambridge, MA 02138, USA
}

\begin{abstract}
The potential of the pure Yang-Mills theory when quantized on the space of gauge fields modulo gauge transformations is computed. The large- $N$ behaviour is given in terms of the Green's function for a scalar field in the adjoint representation.
\end{abstract}

\section{Introduction}

In a pure $\mathrm{SU}(N)$ Yang-Mills theory there are only two inherent parameters in which to expand: the coupling constant $g$ and the group size $N$. In the $N \rightarrow \infty$ limit the perturbative quantum theory is known to simplify in the sense that expectation values of products of equal-time Wilson loop functionals factorize [1]. A mechanism for this reduction has recently been found and applied to simpler field theories with a large- $N$ limit [2]. It is known as the collective-coordinate method and revolves around the fact that as $N$ increases, the symmetry of theory increases. If only states which are invariant under the symmetry group (the group of local gauge transformations for Yang-Mills) are to be considered, then the collective coordinates are to parametrize group-inequivalent fields. When the Hamiltonian is written in terms of the collective coordinates an extra term appears, called the collective-coordinate potential (CCP). In the simpler models studied so far [3], as $N$ increases and the coupling constants are suitably scaled the total potential is found to become sharply peaked around one point. Then the ground-state wave function will be peaked at this point and the expectation values of products of functions of the collective coordinates will factorize. In the case of large- $N$ YangMills such a point of peaking was termed the "master field" by Witten [1].

We attempt to give an intrinsic expression for the CCP in the sense that it depends directly on gauge-invariant properties for the gauge field and not on collective coordinates. More specifically, our expressions (47) and (49) for the total potential in the large- $N$ limit involve only the covariant derivative of the Green's function for an adjoint scalar in a background field. The expression (39) for the CCP is exact for all $N$. These formulas may be more computationally tractable than previous expressions. 
In Sect. 2 the expression for the CCP is derived and discussed. Section 3 has a brief exposition of the geometry of the space of gauge-inequivalent gauge fields. Section 4 gives the computation of the CCP for Yang-Mills, and the large- $N$ behaviour is considered in Sect. 5. The results are discussed in Sect. 6. The geometric proofs of Sect. 2 are relegated to the Appendix.

\section{The General Collective-Coordinate Potential (CCP)}

Suppose one wants to solve a quantum mechanics problem with a potential $V(p)$ on a (possibly) curved space $P$, equipped with a Riemannian metric $g$. Letting $\Delta_{P}$ denote the Laplacian on $P\left(\right.$ given in coordinates by $\left.\Delta_{P} f=\frac{1}{\sqrt{g}}\left(\sqrt{g} g^{\mu \nu} f,{ }_{\nu}\right), \mu\right)$, we
have

$$
-\frac{\hbar^{2}}{2} \Delta_{P} \psi(p)+V(p) \psi(p)=i \hbar \frac{\partial}{\partial t} \psi(p) .
$$

(There is a possible term coming from the scalar curvature of $P$, but this will not arise in the following applications.) In addition suppose that there is a Lie group $G$ which acts freely on $P$ (i.e. if $x \in G$ sends any point of $P$ to itself then $x$ is the identity), acts by isometries (i.e. preserves the metric $g$ ) and leaves $V$ invariant. One may just want to consider wave functions which are $G$-invariant, such as the ground state. Then $\psi$ can be specified equally well by giving its values on the space $M=P / G$ of orbits of $P$ under $G$. The space $M$ has a natural induced metric $\tilde{g}$ given as follows: let $m$ be a point in $M$ (i.e. an orbit in $P$ ) and let $\tilde{A}_{m}, \widetilde{B}_{m}$ be tangent vectors in $M$ at $m$ (i.e. infinitesimal changes in the orbit). Pick a point $p \in P$ in the orbit $m$ and let $X_{p}$ denote the tangent space at $p$ to the orbit $m$. There are unique tangent vectors $A_{p}$ and $B_{p}$ in $P$ at $p$ which are orthogonal to $X_{\tilde{\tau}}$ and give the same infinitesimal change in orbit as $\widetilde{A}_{m}$ and $\widetilde{B}_{m}$. Define $\tilde{g}\left(\tilde{A}_{m}, \widetilde{B}_{m}\right)$ as $g\left(A_{p}, B_{p}\right)$. This is independent of the point $p$ chosen and so is well-defined.

Because the Laplacian commutes with isometries, $\Delta_{P} \psi$ is also a $G$-invariant function and comes from a function $\overline{\Delta_{P} \psi}$ on $M$. There is also the function $\Delta_{M} \psi$ which is the Laplacian of $\psi$ on $M$ using the $\tilde{g}$ metric. These two functions are related in the following way. Let $w^{1 / 2}(m)$ be the relative volume of the orbit $m$, which is defined more precisely in Appendix A. Then

$$
(\tilde{\psi})^{-1} \widetilde{\Delta_{p} \psi}=\left(w^{1 / 4} \tilde{\psi}\right)^{-1} \Delta_{M}\left(w^{1 / 4} \tilde{\psi}\right)-w^{-1 / 4} \Delta_{M} w^{1 / 4} .
$$

For the proof of (2) see Appendix A.

Let $\phi(m)$ be $w^{1 / 4}(m) \tilde{\psi}(m)$. Using (2), (1) becomes

$$
-\frac{\hbar^{2}}{2} \Delta_{M} \phi(m)+(V+\mathrm{CCP})(m) \phi(m)=i \hbar \frac{\partial}{\partial t} \phi(m),
$$

with

$$
\mathrm{CCP}(m)=\frac{\hbar^{2}}{2}\left(w^{-1 / 4} \Delta_{M} w^{1 / 4}\right)(m)
$$

This is the collective-coordinate potential. 
As an example of how this works, let $P$ be $\mathbb{R}^{N}$ without the origin and let $g$ be the Euclidean metric on $P$. Take $G$ to be $\mathrm{SO}(N)$ acting by matrix multiplication on $\mathbb{R}^{N}$ (although this is strictly only a free action when $N=2$ ). Then by sending an orbit to its radius $r, M$ can be identified as the positive real numbers and $g$ is the Euclidean metric on $\mathbb{R}^{+}$. The relative volume of an orbit, $w^{1 / 2}(r)$, is $r^{N-1}$ and

$$
\mathrm{CCP}(r)=\frac{\hbar^{2}}{2} r-\left(\frac{N-1}{2}\right)\left(\frac{N-1}{r}\right)^{\prime \prime}=(N-1)(N-3) \frac{\hbar^{2}}{8 r^{2}} .
$$

This agrees with the previously derived result for the $\mathrm{O}(N)$ linear model [6].

For computational ease, CCP can be written differently. If $\psi=w^{-1 / 4}$ is inserted into (2) then it becomes

$$
w^{1 / 4} \Delta_{P} w^{-1 / 4}=-w^{1 / 4} \Delta_{M} w^{1 / 4} .
$$

Also,

$$
\frac{1}{f} \Delta f=\Delta \ln f+|\nabla \ln f|^{2}
$$

and so

$$
\begin{aligned}
\mathrm{CCP} & =\frac{\hbar^{2}}{8}\left(\Delta_{M} \ln w+\frac{1}{4}\left|\nabla_{M} \ln w\right|^{2}\right) \\
& =\frac{\hbar^{2}}{8}\left(\Delta_{P} \ln w-\frac{1}{4}\left|\nabla_{P} \ln w\right|^{2}\right) .
\end{aligned}
$$

\section{The Reduced Space of Gauge Fields}

In order to apply the preceding to $\mathrm{SU}(N)$ Yang-Mills one must study the space of gauge fields. This has been done by Singer and others [7] and a synopsis of their results will be given. For notation, let $Z$ be the space on which the gauge fields live (such as a torus), let $\Lambda^{0} \otimes \mathrm{SU}(N)$ denote the $\mathrm{SU}(N)$-valued functions on $Z$, and let $\Lambda^{1} \otimes \mathrm{SU}(N)$ denote the $\mathrm{SU}(N)$-valued 1-forms on $Z$ (i.e. vector potentials of the form $\sum_{\mu} A_{\mu}(x) d x^{\mu}$ with $\left.A_{\mu} \in \mathrm{SU}(N)\right)$. Let $z_{\infty}$ be a fixed point on $Z$ (to be thought of as the point at $\infty$ ) and let $G$ be the group of functions $\phi$ from $Z$ to $S U(N)$ such that $\phi\left(z_{\infty}\right)=I$. These act by gauge transformations on $\Lambda^{1} \otimes \mathrm{SU}(N)$ as

$$
\phi \cdot \sum_{\mu} A_{\mu} d x^{\mu}=\sum_{\mu}\left(\phi^{-1} A_{\mu} \phi+\phi^{-1} \partial_{\mu} \phi\right) d x^{\mu} .
$$

Let $D_{A}: \Lambda^{0} \otimes \mathrm{SU}(N) \rightarrow \Lambda^{1} \otimes \mathrm{SU}(N)$ be the covariant derivative: $D_{A} \tau=\sum_{\mu}\left(\partial_{\mu} \tau\right.$ $\left.+\left[A_{\mu}, \tau\right]\right) d x^{\mu}$ and let $D_{A}^{*}: \Lambda^{1} \otimes \mathrm{SU}(N) \rightarrow \Lambda^{0} \otimes \mathrm{SU}(N)$ be its adjoint: $D_{A}^{*}\left(\sum_{\mu} \eta_{\mu} d x^{\mu}\right)$ $=-\sum_{\mu}\left(\partial_{\mu} \eta^{\mu}+\left[A_{\mu}, \eta^{\mu}\right]\right)$. Let $b_{A}: \Lambda^{0} \otimes \mathrm{SU}(N) \rightarrow \Lambda^{1} \otimes \mathrm{SU}(N)$ be the bracket operation: $b_{A} \tau=\sum_{\mu}\left[A_{\mu}, \tau\right] d x^{\mu}$ and let $b_{A}^{*}: \Lambda^{1} \otimes \mathrm{SU}(N) \rightarrow \Lambda^{0} \otimes \mathrm{SU}(N)$ be its adjoint: $b_{A}^{*} \eta$ 
$=-\sum_{\mu}\left[A_{\mu}, \eta^{\mu}\right]$. For $\tau \in \Lambda^{0} \otimes \mathrm{SU}(N)$ we also let $b_{\tau}$ denote bracketing on $\Lambda^{0} \otimes \mathrm{SU}(N)$ or $\Lambda^{1} \otimes \mathrm{SU}(N)$ by components $b_{\tau} \sigma=[\tau, \sigma]$ and $b_{\tau}\left(\sum A_{\mu} d x^{\mu}\right)$ $=\sum_{\mu}\left[\tau, A_{\mu}\right] d x^{\mu}$. A tangent vector $V \in T_{A} \mathfrak{A}$ at a point $A \in \mathfrak{A}$ is given by an infinitesimal change in gauge field, that is, an element $B$ of $\Lambda^{1} \otimes \mathrm{SU}(N)$ such that if $f$ is a differentiable function on $\mathfrak{A},(V f)(A)=\frac{d}{d t} f(A+\varepsilon B)$. If $B, C \in T_{A} \mathfrak{A}$ there is a natural inner product given by

$$
g(B, C)=\int_{Z} \sum_{\mu} \sum_{i, j}\left(B_{\mu}\right)_{i j}^{*}(x)\left(C^{\mu}\right)_{i j}(x) d x .
$$

This makes $\mathfrak{A}$ into an infinite-dimensional flat Riemannian space with a metric invariant under the group $\mathscr{G}$ of gauge transformations. By identifying gaugeequivalent fields one obtains the geodesically complete space $\mathscr{M}=\mathfrak{U} / \mathscr{G}$ of gaugeinequivalent fields with the induced metric. This projection $\pi: \mathfrak{A} \rightarrow \mathscr{M}$ defines a principal $\mathscr{G}$-bundle and the base manifold $\mathscr{M}$ is not flat [7]. We need the relative volumes of orbits of $\mathfrak{A}$ under $\mathscr{G}$. The Lie algebra $g$ of $\mathscr{G}$ is $\left\{\tau \in \Lambda^{0} \otimes \mathrm{SU}(N)\right.$ : $\left.\tau\left(z_{\infty}\right)=0\right\}$. If $R, S \in g$, define their inner product to be

$$
\langle R, S\rangle=\int_{Z} \sum_{i, j} R_{i j}^{*}(x) S_{i j}(x) d x .
$$

At a point $A \in \mathfrak{A}$ the infinitesimal gauge transformation given by $R$ is $A \rightarrow A+\varepsilon D_{A} R$. Using the notation of the appendix, $T_{A}: g \rightarrow g$ is defined by

$$
\left\langle R, T_{A} S\right\rangle_{g}=g\left(D_{A} R, D_{A} S\right)=\left\langle R, D_{A}^{*} D_{A} S\right\rangle_{g} .
$$

Thus $w=\left.\operatorname{det} D_{A}^{*} D_{A}\right|_{g}$. This agrees with a lattice calculation by Lovelace [8].

We remark that there is an interesting formula in [8] giving $w$ as a weighted sum of Wilson loop functionals over the lattice. It was pointed out to me by Singer that without conditions at $\infty$ there is a perfectly well-defined continuum version of this formula. Let $\mu_{x, t}(\gamma)$ be the Wiener measure on the space $\Gamma_{x, t}$ of paths in $Z$ starting at $x$ at time 0 and ending at $x$ at time $t$.

$$
\text { [Formally, } d \mu_{x, t}(\gamma)=\exp \left(-\frac{1}{2} \int_{0}^{t}\left|\frac{d \gamma}{d \sigma}\right|^{2} d \sigma\right) \mathscr{D} \gamma_{x, t} \text { with } \mathscr{D} \gamma_{x, t} \text { as the formal }
$$

measure on such paths $\gamma$. Then if $\chi$ is the character on $\mathrm{SU}(N)$ corresponding to the adjoint representation

$$
\ln w=-\lim _{s \rightarrow 0} \int_{Z} \int_{0}^{\infty} \int_{\Gamma_{x, t}} t^{s-1} \chi\left(P \exp \left(-\oint_{\gamma} A_{v}(\sigma) d \sigma^{v}\right)\right) d \mu_{x, t} d t d x \quad[13] .
$$

\section{Application to Yang-Mills Fields}

The CCP for Yang-Mills can be derived by writing the quantum Yang-Mills equations in a Schrödinger-type form [9]. Let the spacetime be $\mathbb{R} \times T^{3}$ with $T^{3}$ a finite volume 3-torus and let the metric be the flat $(+---)$ Lorentz metric. The following can be considered as the continuum limit of the Kogut-Susskind lattice 
Hamiltonian. For the Yang-Mills Lagrangian

$$
\begin{gathered}
\mathscr{L}=\frac{1}{2 g^{2}} \sum_{\mu<\nu} \operatorname{Tr} F_{\mu \nu} F^{\mu \nu}, \\
F_{\mu \nu}=\partial_{\mu} A_{\nu}-\partial_{\nu} A_{\mu}+\left[A_{\mu}, A_{\nu}\right],
\end{gathered}
$$

we pick the local gauge condition $A_{0}=0$ to derive the Hamiltonian,

$$
H=\frac{1}{2} g^{2} \int_{Z} \sum_{i, a} \pi_{i a}(x) \pi_{i a}(x) d^{3} x-\frac{1}{2 g^{2}} \int_{Z} \sum_{i<j} \operatorname{Tr} F_{i j}(x) F^{i j}(x) d^{3} x,
$$

with the Gaussian constraint

$$
\sum_{i} D_{i} \pi^{i}=\sum_{i}\left(\partial_{i} \pi^{i}+\left[A_{i}, \pi^{i}\right]\right)=0 .
$$

(Here $1 \leqq i, j \leqq 3$ and $a$ is a Lie algebra index.) Hereafter, we use the positive definite Euclidean metric on $Z=T^{3}$. The commutation relations

$$
\left[\pi_{a}^{i}(x), A_{b}^{j}(y)\right]=-i \hbar \delta^{i j} \delta^{3}(x-y) \delta_{a b}
$$

can be formally represented by

$$
\pi_{a}^{i}(x)=-i \frac{\delta}{\delta A_{a}^{i}(x)}
$$

then as an operator on a function $f$ on $\mathfrak{A}, H$ becomes

$$
(H f)(A)=-\frac{\hbar^{2} g^{2}}{2}(\Delta f)(A)+\frac{1}{2 g^{2}}\|F(A)\|^{2} f(A),
$$

with

$$
(\Delta f)(A)=\int_{Z} \sum_{i, a} \frac{\delta}{\delta A_{i a}(x)^{2}} d^{3} x
$$

and

$$
\|F(A)\|^{2}=-\int_{Z} \sum_{i<j} \operatorname{Tr} F_{i j}^{2}(x) d^{3} x .
$$

This Laplacian is the same as the geometric Laplacian on the space of irreducible gauge fields on $T^{3}$ and the Gaussian constraint $D_{i} \frac{\delta f}{\delta A_{i}(x)}$ says that $f$ is a gaugeinvariant function on $\mathfrak{A}$. We can apply (3) to conclude that as operators on functions on $\mathscr{M}$,

$$
-\frac{1}{2} \hbar^{2} g^{2} \Delta_{\mathscr{M}} \phi+\left(\frac{1}{2 g^{2}}\|F\|^{2}+\mathrm{CCP}\right) \phi=i \hbar \frac{\partial \phi}{\partial t}
$$

with $w=\left.\operatorname{det} D_{A}^{*} D_{A}\right|_{g}, \phi=w^{1 / 4} f$ and

$$
\mathrm{CCP}=\frac{\hbar^{2} g^{2}}{8}\left(\Delta_{\mathfrak{U}} \ln w-\frac{1}{4}\left|\nabla_{\mathfrak{N}} \ln w\right|^{2}\right)
$$


If suitably regularized the terms in CCP can be computed. For a trace class operator $T$, let $\operatorname{Sp}(T)$ be the trace of $T$. If $T$ can be represented by $(T f)(X)$ $=\int K(X, Y) f(Y) d Y$ for a continuous kernel $K(X, Y)$, we have $\operatorname{Sp}(T)$ $=\int K(X, X) d X$. Let $G_{A}$ be $\left(D_{A}^{*} D_{A}\right)^{-1}$, the background-field Green's function for a scalar in the adjoint representation. Let $\left\{\varrho_{j}\right\}_{j=1}^{N^{2}-1}$ be an orthonormal basis for $\mathrm{SU}(N)$ under the Killing-form inner product, with $\left[\varrho_{j}, \varrho_{k}\right]=c_{j k l} \varrho_{l}$. Let $\left\{f_{j}\right\}_{j=1}^{\infty}$ be an orthonormal basis for $\Lambda^{0} \otimes \operatorname{SU}(N)$ consisting of eigenvectors of $D_{A}^{*} D_{A}$ with eigenvalues $\left\{\lambda_{j}\right\}_{j=1}^{\infty}$.

In order to treat $D_{A}^{*} D_{A}$ acting on $g$ we must first restrict the domain to functions which are sufficiently continuous to have a value at $z_{\infty}$, by introducing Sobolev spaces (see Appendix B). The effect of using $g$ instead of $\Lambda^{0} \otimes \operatorname{SU}(N)$ can then be subtracted from all determinant calculations. For brevity we will only show the calculations on $\Lambda^{0} \otimes \mathrm{SU}(N)$, as the subtracted terms will go away in the large volume limit. The variation of $D_{A}^{*} D_{A}$ under a variation of $A$ is

$$
\left.\frac{d}{d \varepsilon}\right|_{\varepsilon=0}\left(D^{*} D\right)_{A+\varepsilon \eta}=b_{\eta}^{*} D_{A}+D_{A}^{*} b_{\eta} .
$$

The method of zeta-function regularization [10] gives

$$
\begin{aligned}
\left.\frac{d}{d \varepsilon}\right|_{\varepsilon=0} \ln \operatorname{det}\left(D^{*} D\right)_{A+\varepsilon \eta} & =\lim _{s \rightarrow 0} \operatorname{Sp}\left(\left(b_{\eta}^{*} D_{A}+D_{A}^{*} b_{\eta}\right) G_{A}^{s+1}\right) \\
& =\lim _{s \rightarrow 0} \sum_{j}\left\langle f_{j},\left(b_{\eta}^{*} D_{A}+D_{A}^{*} b_{\eta}\right) G_{A}^{(s+1)} f_{j}\right\rangle \\
& =\lim _{s \rightarrow 0} 2 \sum_{j}\left\langle f_{j}, b_{\eta}^{*} D_{A} G_{A}^{(s+1)} f_{j}\right\rangle \\
& =\left\langle\eta, E_{A}\right\rangle
\end{aligned}
$$

with $E_{A} \in \Lambda^{1} \otimes \mathrm{SU}(N)$ given by

$$
E_{A}=\lim _{s \rightarrow 0} 2 \sum_{i, j}\left[f_{j},\left(D_{A}\right)_{i} G_{A}^{(s+1)} f_{j}\right] d x^{i} .
$$

Although, this only literally exists for $\operatorname{Re}(s)>\frac{1}{2}$, it can be analytically continued to give a finite value at $s=0$.

For the second variation of $\ln w$, we have

$$
\begin{aligned}
\left.\frac{d^{2}}{d \varepsilon^{2}}\right|_{\varepsilon=0} \ln \operatorname{det}\left(D^{*} D\right)_{A+\varepsilon \eta}=\left.\lim _{s \rightarrow 0} \operatorname{Sp} \frac{d}{d \varepsilon}\right|_{\varepsilon=0}\left(\left(b_{\eta}^{*} D_{A+\varepsilon \eta}+D_{A+\varepsilon \eta}^{*} b_{\eta}\right) G_{A+\varepsilon \eta}^{(s+1)}\right) \\
=\lim _{s \rightarrow 0} \operatorname{Sp}\left(2 b_{\eta}^{*} b_{\eta} G_{A}^{(s+1)}-\frac{1}{\Gamma(s+1)} \int_{0}^{\infty} t^{s} \int_{0}^{t}\left(b_{\eta}^{*} D_{A}+D_{A}^{*} b_{\eta}\right)\right. \\
\left.\cdot e^{-V D_{A}^{*} D_{A}}\left(b_{\eta}^{*} D_{A}+D_{A}^{*} b_{\eta}\right) e^{(V-t) D_{A}^{*} D_{A}} d V d t\right) .
\end{aligned}
$$

The first term of (29) is

$$
\begin{aligned}
\operatorname{Sp}\left(2 b_{\eta}^{*} b_{\eta} G_{A}^{(s+1)}\right) & =\sum_{j}\left\langle f_{j}, 2 b_{\eta}^{*} b_{\eta} G_{A}^{(s+1)} f_{j}\right\rangle \\
& =\left\langle\eta,-2 \sum_{j} \lambda_{j}^{-(s+1)} b_{f_{j}} b_{f_{j}} \eta\right\rangle .
\end{aligned}
$$


Using the identity

$$
\left(b_{\eta}^{*} D_{A}+D_{A}^{*} b_{\eta}\right) f_{j}=-\left(2 b_{D_{A} f_{j}}^{*}+b_{f_{j}} D_{A}^{*}\right) \eta,
$$

the second term of (29) is

$$
\begin{aligned}
& -\frac{1}{\Gamma(s+1)} \int_{0}^{\infty} t^{s} \int_{0}^{t}\left\langle\eta, \sum_{j} e^{(V-t) \lambda_{j}}\left(2\left[b_{f_{J}^{*}}, D_{A}\right]+D_{A} b_{f_{j}^{*}}\right)\right. \\
& \left.\cdot e^{-V D_{A}^{*} D_{A}}\left(2\left[D_{A}^{*}, b_{f_{j}}\right]+b_{f_{j}} D_{A}^{*}\right) \eta\right\rangle \\
= & \left\langle\eta, \sum_{j}\left(2 b_{D_{A} f_{j}}+D_{A} b_{f_{j}^{*}}\right)\left(\lambda_{j}^{-(s+1)}-G_{A}^{(s+1)}\right)\left(\lambda_{j}-D_{A}^{*} D_{A}\right)\left(2 b_{D_{A} f_{j}}^{*}+b_{f_{j}} D_{A}^{*}\right) \eta\right\rangle .
\end{aligned}
$$

Thus we can write (29) as

$$
\left.\frac{d^{2}}{d \varepsilon^{2}}\right|_{\varepsilon=0} \ln \operatorname{det}\left(D^{*} D\right)_{A+\varepsilon \eta}=\left\langle\eta, \theta_{A} \eta\right\rangle
$$

with

$$
\begin{aligned}
\theta_{A}= & \lim _{s \rightarrow 0} \sum_{j}\left\{-2 \lambda_{j}^{-(s+2)} b_{f_{j}} b_{f_{j}}+\left(2 b_{D_{A} f_{j}}+D_{A} b_{f_{j}^{*}}\right)\right. \\
& \left.\cdot\left(\lambda_{j}^{-(s+1)}-G_{A}^{(s+1)}\right)\left(\lambda_{j}-D_{A}^{*} D_{A}\right)^{-1}\left(2 b_{D_{A}}^{*} f_{j}+b_{f_{j}} D_{A}^{*}\right)\right\} .
\end{aligned}
$$

This is a well-defined self-adjoint second-order pseudo-differential operator $\left(\psi d 0_{2}\right)[11]$.

The Laplacian $\Delta_{\mathfrak{U}} \ln w$ is $\operatorname{Sp}\left(\theta_{A}\right)$; then

$$
\mathrm{CCP}=\frac{\hbar^{2} g^{2}}{8}\left(\operatorname{Sp}\left(\theta_{A}\right)-\frac{1}{4}\left\langle E_{A}, E_{A}\right\rangle\right) .
$$

Using (8) we can write CCP to make the trace act on a nicer operator than $\theta_{A}$. Let $\left\{\sigma_{k}\right\}_{k=1}^{\infty}$ be an orthonormal basis of $\operatorname{Ker} D_{A}^{*}$. Because the geodesics in $\mathscr{M}$ are projections of geodesics in $\mathfrak{A}$,

$$
\begin{aligned}
\Delta_{\mathcal{M}} \ln \operatorname{det} D^{*} D= & \left.\sum_{k} \frac{d^{2}}{d \varepsilon^{2}}\right|_{\varepsilon=0} \ln \operatorname{det}\left(D^{*} D\right)_{A+\varepsilon \sigma_{k}} \\
= & \sum_{k}\left\langle\sigma_{k}, \theta_{A} \sigma_{k}\right\rangle \\
= & \sum_{k}\left\langle\sigma_{k}, \lim _{s \rightarrow 0} \sum_{j}\left\{-2 \lambda_{j}^{-(s+2)} b_{f_{j}} b_{f_{j}}+4 b_{D_{A} f_{j}}\right.\right. \\
& \left.\left.\cdot\left(\lambda_{j}^{-(s+1)}-G_{A}^{(s+1)}\right)\left(\lambda_{j}-D_{A}^{*} D_{A}\right)^{-1} b_{D_{A} f_{j}}^{*}\right\} \sigma_{k}\right\rangle .
\end{aligned}
$$

Because the projection operator on $\operatorname{Ker} D_{A}^{*}$ is $I-D_{A} G_{A} D_{A}^{*}$, we have

$$
\Delta_{\mathscr{M}} \ln w=\operatorname{Sp}\left(\mathscr{U}_{A}\right)
$$

with

$$
\begin{aligned}
\mathscr{U}_{A}= & \lim _{s \rightarrow 0}\left(I-D_{A} G_{A} D_{A}^{*}\right) \sum_{j}\left\{-2 \lambda_{j}^{-(s+2)} b_{f_{j}} b_{f_{j}}\right. \\
& \left.+4 b_{D_{A} f_{j}}\left(\lambda_{j}^{-(s+1)}-G_{A}^{(s+1)}\right)\left(\lambda_{j}-D_{A}^{*} D_{A}\right)^{-1} b_{D_{A} f_{j}}^{*}\right\}\left(I-D_{A} G_{A} D_{A}^{*}\right) .
\end{aligned}
$$


This is a $\psi d 0_{0}$. Then

$$
\mathrm{CCP}=\frac{h^{2} g^{2}}{8}\left(\operatorname{Sp}\left(\mathscr{U}_{A}\right)+\frac{1}{4}\left\langle E_{A}, E_{A}\right\rangle\right)
$$

\section{The Large- $N$ Limit}

The pure $\mathrm{SU}(N)$ Yang-Mills theory has a large- $N$ limit in the sense that if $g^{2} N$ is fixed then for any loop $L$,

$$
W_{L}(A)=\left\langle\frac{1}{N} \operatorname{Tr} P \exp \left(-\oint_{L} A \cdot d s\right)\right\rangle
$$

approaches a limit as $N \rightarrow \infty$. We can think of $\left\{W_{L}\right\}$ as coordinates for a linear space $\mathscr{W}$ with the manifold $\mathscr{M}_{N}$ of $\mathrm{SU}(N)$ gauge-inequivalent gauge fields embedded in $\mathscr{W}$ as $\phi_{N}\left(\mathscr{M}_{N}\right)$ by sending $A \in \mathscr{M}_{N}$ to $\phi_{N}(A)=\left\{W_{L}(A)\right\} \in \mathscr{W}$. For $A \in \mathscr{M}_{N}$, the $\mathrm{SU}(p N)$ field

$$
\left.\tilde{A}=\left(\begin{array}{cccc}
A & 0 & \ldots & 0 \\
0 & A & \ldots & 0 \\
\vdots & \vdots & & \vdots \\
0 & 0 & \ldots & A
\end{array}\right)\right\} p N
$$

in $\mathscr{M}_{p N}$ has $W_{L}(\tilde{A})=W_{L}(A)$ for all $L$; if $\left\{N_{i}\right\}_{i=1}^{\infty}$ is a sequence with $N_{i} \mid N_{i+1}$ then $\phi_{N_{1}}\left(\mathscr{M}_{N}\right) \rightarrow \phi_{N_{2}}\left(\mathscr{M}_{N_{2}}\right) \rightarrow \ldots \rightarrow \phi_{N_{i}}\left(\mathscr{M}_{N_{i}}\right) \rightarrow \ldots$ gives a chain of submanifolds of $\mathscr{W}$. If $\tilde{x} \in \bigcup_{i} \phi_{N_{i}}\left(\mathscr{M}_{N_{i}}\right)$, then the leading term of $(V+\mathrm{CCP})_{N_{i}}\left(\phi_{N_{i}}^{-1}(\tilde{x})\right)$ in $i$ should dominate in the large- $N$ limit.

There has been confusion in the literature on the role of the kinetic-energy term in the large- $N$ limit. In the Hermitian matrix model the total potential vanishes and the large- $N$ is given by constraints on the wave function from the geodesic incompleteness of the base manifold $\mathscr{M}$ [5]. In our case the base manifold is geodesically complete and we conjecture that Yang-Mills is similar to the $\mathrm{O}(N)$ linear model in that the kinetic contribution will be nonleading order in $N$.

Lovelace has speculated that only the $\left|\nabla_{\mathscr{M}} \ln w\right|^{2}$ term of (8) contributes for large $N$ [8]. Before showing this we must make a regularization of the trace in (39). First, suppose that $M_{F}$ is the operator for multiplication by a continuous function $F$ on $L^{2}(Z)$. To define $\operatorname{Sp}\left(M_{F}\right)$ suppose that $Z$ is latticed by $l$ points in $Z$. Then for large $l$, $\operatorname{Sp}\left(M_{F}\right)$ as defined on the lattice is $l \int_{Z} F(x) d x / \operatorname{vol}(Z)$. In order to extend this definition to other $\psi d 0_{0}$ 's, let $a(z, \xi)$ be the symbol of $\mathscr{U}_{A}$, so

$$
\left(\mathscr{U}_{A} f\right)(z)=\frac{1}{(2 \pi)^{3}} \iint e^{i(z-y) \cdot \xi} a(z, \xi) f(y) d y d \xi .
$$

If $a_{0}$ is the zeroth order part of $a$, we define

$$
\text { "Sp" }\left(\mathscr{U}_{A}\right)=l \int_{S^{*} Z} a_{0}(z, \xi) d z d \xi / \operatorname{vol}\left(S^{*} Z\right),
$$

where $S^{*} Z$ is the cosphere bundle $\left\{(z, \xi) \in T^{*} Z:|\xi|^{2}=1\right\}$. 
This regularizes the most singular part of $\operatorname{Sp}\left(\mathscr{U}_{A}\right)$ in terms of a lattice spacing. Computing $a_{0}$ from (38) and using the fact that "Sp" is tracial, we obtain

$$
\text { "Sp" }\left(\mathscr{U}_{A}\right)=-\frac{4}{3} \frac{l}{\operatorname{vol}(Z)} \lim _{s \rightarrow 0} \operatorname{Sp} G_{A}^{(s+1)} .
$$

From (39),

$$
\mathrm{CCP}=\frac{\hbar^{2} g^{2}}{8}\left(-\frac{4}{3} \frac{l}{\operatorname{vol}(Z)} \lim _{s \rightarrow 0} \operatorname{Sp} G_{A}^{(s+1)}+\frac{1}{4}\left\langle E_{A}, E_{A}\right\rangle\right) .
$$

For a fixed $\tilde{x} \in \bigcup_{i} \phi_{N_{i}}\left(\mathscr{M}_{N_{i}}\right)$, let $A_{i}(\tilde{x})$ be $\phi_{N_{i}}^{-1}(\tilde{x})$ (this exists for large enough $i$ ). Clearly, $\left\|F\left(A_{i}(\tilde{x})\right)\right\|^{2} \sim N_{i}$. The eigenvalues of $\left(D^{*} D\right)_{A}$ acting on $g$ consist of $p^{2}$ copies of the eigenvalues of $\left(D^{*} D\right)_{A}$; thus $\lim _{s \rightarrow 0} \operatorname{Sp} G_{A_{i}(\tilde{x})}^{(s+1)} \sim N_{i}^{2}$. Because $E_{\tilde{A}}$ is made of copies of $p \cdot E_{A}$ strung along the diagonal, $\left\langle E_{A_{i}(\tilde{x})}, E_{A_{i}(\tilde{x})}\right\rangle \sim N_{i}^{3}$. Then

$$
\begin{gathered}
\frac{1}{2 g^{2}}\left\|F\left(A_{i}(x)\right)\right\|^{2} \sim N_{i}^{2}, \\
\frac{\hbar^{2} g^{2}}{8}\left(-\frac{4}{3} \frac{l}{\operatorname{vol}(Z)} \lim _{s \rightarrow 0} \operatorname{Sp} G_{A_{i}(\tilde{x})}^{(s+1)}\right) \sim N_{i},
\end{gathered}
$$

and

$$
\frac{\hbar^{2} g^{2}}{8}\left(\frac{1}{4}\left\langle E_{A_{i}(\tilde{x})}, E_{A_{i}(\tilde{x})}\right\rangle\right) \sim N_{i}^{2} .
$$

It is in this sense that in the large- $N$ limit the dominating terms in the total potential are

$$
\frac{1}{2 g^{2}}\|F(A)\|^{2}+\frac{\hbar^{2} g^{2}}{32}\left\langle E_{A}, E_{A}\right\rangle
$$

To write $E_{A}$ more explicitly, define the $\operatorname{kernel}\left(\left(D_{A}\right)_{i} G_{A}^{(s+1)}\right)(x, y)$ of $D_{A} G_{A}^{(s+1)}$ by

$$
\left.\left(\left(\left(D_{A}\right)_{i} G_{A}^{(s+1)}\right)\left(f \otimes \varrho_{p}\right)\right)(x)=\sum_{q} \int_{Z}\left(D_{A}\right)_{i} G_{A}^{(s+1)}\right)_{q p}(x, y) f(y) d y \otimes \varrho_{q} .
$$

Then

$$
E_{A}=\lim _{s \rightarrow 0} \sum_{p, q, r} 2 c_{p q r}\left(\left(D_{A}\right)_{i} G_{A}^{(s+1)}\right)_{q p}(x, x) d x^{i} \otimes \varrho_{r}
$$

\section{Remarks}

It has been shown that the name "collective-coordinate potential" is a misnomer in that the CCP can be evaluated in a wholly coordinate-free manner. The large- $N$ total potential (47) is finite and smooth, a property due to the fact that when doing an $n+1$ dimensional Yang-Mills theory with $n$ odd, the regularized Green's 
function will exist and be smooth when taken from a point to itself. For even $n$ there will generally be a pole in $s$ when computing $\lim _{s \rightarrow 0} G_{A}^{(s+1)}(x, x)$.

A collective-coordinate potential was previously computed by Sakita using Wilson loop functionals as coordinates for $\mathscr{M}$ [4]. Our expression differs from his because of different kinetic energy terms on $\mathscr{M}$. Sakita uses $-\frac{\hbar^{2}}{2} g^{\mu v} \psi, \mu \nu$, whereas we use the coordinate-invariant expression $-\frac{\hbar^{2}}{2} \frac{1}{\sqrt{g}}\left(\sqrt{g} g^{\mu v} \psi, \mu\right), v$. Thus the potentials will also be different.

It may be possible to extend the preceding methods to other field theories with large symmetry groups, such as gravity and string models. For Yang-Mills, assuming the kinetic energy is nonleading order in $N$, the master field should be obtained by minimizing (47) for large $N$.

\section{Appendix A}

The hypotheses of Sect. 2 essentially define a principal $G$-bundle [12] $\pi: P \rightarrow M$ with a right $G$-invariant metric $g$ on $P$. The Lie algebra $g$ of $G$ has a representation $q$ by vector fields on $P$ given by sending an $x \in g$ to the vector field,

$$
q(X)_{p}=\left.\frac{d}{d T}\right|_{T=0}\left(p \cdot e^{T X}\right) .
$$

Let $\langle,\rangle_{g}$ be any inner product on $g$. For a point $p \in P$, define a linear transformation $T_{p}$ by

$$
\left\langle X, T_{p} Y\right\rangle=g\left(q(X)_{p}, q(Y)_{p}\right), \text { where } X, Y \in g .
$$

Then $w(p)$ is defined as $\operatorname{det} T_{p}$. This is a $G$-invariant function and so can be considered as a function on the space of orbits $M$.

Proposition. If $f$ is a G-invariant $C^{2}$ function on $P$, then

$$
f^{-1} \Delta_{p} f=\left(w^{1 / 4} f\right)^{-1} \Delta_{M}\left(w^{1 / 4} f\right)-w^{-1 / 4} \Delta_{M} w^{1 / 4} .
$$

Proof. At a point $p \in P$, define the vertical space $\mathbb{V}_{P}$ to be the tangent space of the $G$-orbit at $p$, i.e. $\operatorname{span}\left(q(X)_{p}\right)$. Define the horizontal space $\mathbb{H}_{p}$ to be the orthogonal complement $\mathbb{V}_{p}^{\perp}$. If $X \in g$ and $R_{e^{T X}}$ denotes the action of $e^{T X}$ on $P$, then for any horizontal vector field $H,\left(R_{e^{-T X}}\right)_{*} H$ is horizontal and

$$
\left.\frac{d}{d t}\right|_{t=0}\left(R_{e^{-T X}}\right)_{*} H_{p e^{t X}}=[q(X), H]_{p}
$$

is horizontal. Let $\left\{e_{i}\right\}$ and $\left\{e_{\alpha}\right\}$ be $G$-invariant orthonormal bases for $\mathbb{V}$ and $\mathbb{H}$, respectively. Then $\left\{\pi_{*} e_{\alpha}\right\}$ is an orthonormal framing of $M$, and if $\tilde{\nabla}, \nabla$ are the LeviCivita connections on $P$ and $M$,

$$
\pi_{*}\left(\tilde{\nabla}_{e_{\alpha}} e_{\beta}\right)=\nabla_{\pi_{*} e_{\alpha}} \pi_{*} e_{\beta} .
$$


We have

$$
\begin{aligned}
\Delta_{P} f & =\sum_{i} e_{i}^{2} f+\sum_{\alpha} e_{\alpha}^{2} f-\sum_{i}\left(\tilde{\nabla_{e_{i}}} e_{i}\right) f-\sum_{\alpha}\left(\tilde{\nabla}_{e_{\alpha}} e_{\alpha}\right) f \\
& =\Delta_{M} f-\sum_{i}\left(\tilde{\nabla_{e_{i}}} e_{i}\right) f \\
& =\Delta_{M} f-\sum_{i, \alpha} g\left(\tilde{\nabla}_{e_{i}} e_{i}, e_{\alpha}\right) e_{\alpha} f \\
& =\Delta_{M} f+\sum_{i, \alpha} g\left(e_{i}, \tilde{\nabla}_{e_{i}} e_{\alpha}\right) e_{\alpha} f \\
& =\Delta_{M} f+\sum_{i, \alpha} g\left(e_{i}, \tilde{\nabla}_{e_{\alpha}} e_{i}+\left[e_{i}, e_{\alpha}\right]\right) e_{\alpha} f \\
& =\Delta_{M} f+\sum_{i, \alpha} g\left(e_{i},\left[e_{i}, e_{\alpha}\right]\right) e_{\alpha} f .
\end{aligned}
$$

Let $\left\{X_{j}\right\}$ be an orthonormal basis for $g$ under \langle\rangle$_{g}$ and let $f_{i}=q\left(X_{j}\right)$. Then if

$$
\begin{aligned}
& e_{i}=\sum_{i} c_{i j} f_{j} \text { and } f_{j}=\sum_{k} d_{j k} e_{k}, \\
& \begin{aligned}
\Delta_{P} f & =\Delta_{M} f+\sum_{i, \alpha} g\left(e_{i},\left[\sum_{j} c_{i j} f_{j}, e_{\alpha}\right]\right) e_{\alpha} f \\
& =\Delta_{M} f+\sum_{i, \alpha} g\left(e_{i}, \sum_{j} c_{i j}\left[f_{j}, e_{\alpha}\right]-\sum_{j}\left(e_{\alpha} c_{i j}\right) f_{j}\right) e_{\alpha} f \\
& =\Delta_{M} f-\sum_{i, \alpha}\left(e_{\alpha} c_{i j}\right) d_{j i}\left(e_{\alpha} f\right) \\
& =\Delta_{M} f-\sum_{\alpha}\left(e_{\alpha} \ln \operatorname{det} c\right)\left(e_{\alpha} f\right)
\end{aligned}
\end{aligned}
$$

Now

$$
\left\langle X_{k}, T_{p} X_{l}\right\rangle=g\left(f_{k}, f_{l}\right)=g\left(\sum_{m} d_{k m} e_{m}, \sum_{n} d_{l n} e_{n}\right)=\sum_{m} d_{k m} d_{l m},
$$

so $T_{p}=d d^{T}$ and

$$
\operatorname{det} c=(\operatorname{det} d)^{-1}=\left(\operatorname{det} T_{p}\right)^{-1 / 2}=w^{-1 / 2} .
$$

If $(,)_{M}$ denotes the inner product on $M$,

$$
\Delta_{P} f=\Delta_{M} f+\frac{1}{2 w}\left(\nabla_{M} w, \nabla_{M} f\right)_{M}
$$

Because

$$
\Delta_{M}\left(w^{1 / 4} f\right)=w^{1 / 4} \Delta_{M} f+\frac{1}{2} w^{-3 / 4}\left(\nabla_{M} w, \nabla_{M} f\right)_{M}+f \Delta_{M} w^{1 / 4}
$$

we have

$$
\begin{aligned}
& \Delta_{p} f=w^{-1 / 4} \Delta_{M}\left(w^{1 / 4} f\right)-w^{1 / 4} f \Delta_{M} w^{-1 / 4} \text { and } f^{-1} \Delta_{p} f=\left(w^{1 / 4} f\right)^{-1} \Delta_{M}\left(w^{1 / 4} f\right) \\
& -w^{-1 / 4} \Delta_{M} w^{1 / 4} \text {. }
\end{aligned}
$$

For a more geometric interpretation of this extra term, let $\mathbf{K}(m)$ be the mean curvature normal to the orbit above $m \in M$, when projected onto $M$. Then

$$
-w^{-1 / 4} \Delta_{M} w^{1 / 4}=\frac{1}{2} \operatorname{div}_{M} \mathbf{K}-\frac{1}{4}(\mathbf{K}, \mathbf{K})_{M} .
$$




\section{Appendix B}

We want to treat the operator $D_{A}^{*} D_{A}$ on the $L^{2}$ space of elements of $\Lambda^{0} \otimes \operatorname{SU}(N)$ vanishing at $z_{\infty}$. However, this condition does not make sense on $L^{2}$ functions; we work instead on Sobolev spaces $H_{T}$ [11]. If $T>\frac{1}{2} \operatorname{dim} Z$ then elements $f, g$ of $H_{T}$ are continuous functions and $\langle f, g\rangle_{H_{T}}=\left\langle f,\left(1-\nabla^{2}\right)^{T} g\right\rangle L^{2}$. Then

$$
\left(D_{A}^{*} D_{A}\right)_{H_{T}}=\left(1-\nabla^{2}\right)^{-T} D_{A}^{*}\left(1-\nabla^{2}\right)^{T} D_{A} \text {. }
$$

Let $V \in H_{T}$ be such that $\langle V, f\rangle_{H_{T}}=f\left(z_{\infty}\right)$; we have $V=\left(1-\nabla^{2}\right)^{-T} \delta_{z_{\infty}}$. Then

$$
\left.\operatorname{Sp}\left(\left(D_{A}^{*} D_{A}\right)_{H_{T}}\right)^{-s}\right|_{g}=\operatorname{Sp}\left(D_{A}^{*} D_{A}\right)_{H_{T}}^{-s}-\frac{\left\langle V\left|\left(D_{A}^{*} D_{A}\right)_{H_{T}}^{-s}\right| V\right\rangle}{\langle V \mid V\rangle_{H_{T}}} .
$$

This extra term can be carried throughout the determinant calculation.

Acknowledgements. I thank I. Singer, K. Bardakci, and D. Gross for helpful discussions.

\section{References}

1. Witten, E.: 1979 Cargese lectures: recent developments in gauge theories. t'Hooft, G. (ed.). New York: Plenum Press 1980

2. Jevicki, A., Sakita, B.: The quantum collective field method and its application to the planar limit. Nucl. Phys. B 165, 511 (1980)

3. Bardakci, K., Caldi, D.G., Neuberger, H.: Dominant Euclidean configurations for all N. Nucl. Phys. B 177, 333 (1981)

4. Sakita, B.: Field theory of strings as a collective field theory of $U(N)$ gauge fields. Phys. Rev. D 21, 1067 (1980)

5. Brézin, E., Itzykson, C., Parisi, G., Zuber, J.B.: Planar diagrams. Commun. Math.Phys. 59, 35 (1978)

6. Bardakci, K.: Classical solutions and the large $N$-limit. Nucl. Phys. B 178, 263 (1981)

7. Singer, I.M.: Some remarks on the Gribov ambiguity. Commun. Math. Phys. 60, 7 (1978); Babelon, O., Viallet, C.M.: The Riemannian geometry of the configuration space of gauge theories. Commun. Math. Phys. 81, 515 (1981) and references therein

8. Lovelace, C.: Construction of the $N=\infty$ master field for strong coupling. Nucl. Phys. B 197, $76(1982)$

9. Feynman, R.P.: The qualitative behavior of Yang-Mills theory in $2+1$ dimensions. Nucl. Phys. B 188, 479 (1981);

Singer, I.M.: The geometry of orbit space for nonabelian gauge theories. Phys. Scri. 24, 817 (1981)

10. Ray, D.B., Singer, I.M.: R-torsion and the Laplacian on Riemannian manifolds. Adv. Math. 7, 145 (1971);

Corrigan, E., Goddard, P., Osborn, H., Templeton, S.: Zeta-function regularization and multi-instanton determinants. Nucl. Phys. B 159, 469 (1979)

11. Seeley, R.: 1968 CIME Lectures: topics in pseudo-differential operators. Edizioni Cremonese, 1969

12. Kobayashi, S., Nomizu, K.: Foundations of differential geometry, Vol. 1, p. 50. New York: Interscience 1963

13. Stroock, D.: On certain systems of parabolic equations. Comm. Pure Appl. Math. 23, 447 (1970)

Communicated by A. Jaffe

Received March 10, 1984; in revised form April 4, 1984 